\title{
LA CONCEPTUALIZACIÓN DEL TIEMPO A TRAVÉS DE LA METÁFORA ESPACIO-TEMPORAL CON EL VERBO LLEGAR
}

\section{CONCEPTUALIZING TIME THROUGH SPACE-TIME METAPHORS: THE CASE OF LLEGAR}

\author{
Alexandra Astrid Ruiz Surget \\ Universidad Autónoma Metropolitana, Doctorado en Lingüística
}

RESUMEN

Este artículo desarrolla un análisis, basado en el modelo de la integración conceptual (Fauconnier \& Turner, 2002), de los rasgos semánticos de las metáforas regidas por el verbo llegar, obtenidas en entrevistas a jóvenes profesionistas de la Ciudad de México pertenecientes al mismo nivel socioeconómico. Como parte de mi investigación doctoral, he llevado a cabo una serie de entrevistas por grupos de edad en busca de las estrategias discursivas de construcción metafórica de marcos conceptuales espacio-temporales que utilizan los individuos para relatar proyectos y episodios de vida. Una de las estrategias, la cual desarrollo en este artículo, se encuentra en el nivel de la construcción semántica del concepto tiempo. Las personas utilizan componentes lingüísticos que codifican espacio, como el verbo llegar, la preposición a y los deícticos locativos, para referirse al parámetro del tiempo e indicar los cambios que ocurren a lo largo de la vida o por el advenimiento de un suceso. Los ejemplos de metáforas que aquí presento son de dos tipos: la forma conjugada del verbo llegar y la perífrasis ir a + infinitivo. Los individuos emplean estas formas ya sea para posicionarse en el discurso como los agentes que realizan la acción de llegar a alguna edad o etapa de vida o bien para indicar la inevitabilidad de la llegada de un suceso. Por lo tanto, en este artículo también explico estas dos formas de conceptualización, siguiendo el trabajo de Boroditsky (2000), quien las denomina subesquemas de una misma metáfora espacio-temporal.

Estudios de Lingüística Aplicada, año 35, número 66, diciembre de 2017, pp. 173-203 doi: 10.22201/enallt.01852647p.2017.66.836 
PALABRAS ClAVE: razonamiento metafórico; integración conceptual; envejecimiento; tiempo-espacio; trayecto de vida

Abstract

This study examines metaphoric expressions using the Spanish verb llegar (to arrive) through the conceptual integration model (Fauconnier \& Turner, 2002). These metaphoric expressions were obtained from interviews to young professionals from the same socioeconomic level who live and work in Mexico City. These interviews were administered, as part of my doctoral research, to various groups of persons stratified by age. The main purpose is to find discursive strategies used by people to metaphorically create spatial-temporal conceptual frameworks in order to recount a project or an episode of their lives. In this study, I drafted one discursive strategy that is observed at a semantic level: the use of spatial linguistic components for referring to time. This is the case of metaphors involving the verb llegar used to narrate the changes that people underwent in their lives or the occurrence of an event. There are two types of examples in this study: metaphors that use a conjugated form of llegar and metaphors that use the periphrasis ir a + infinitivo (going to + infinitive verb). Subjects use these speech modalities to place themselves discursively as agents who perform the actions of reaching a certain age or stage in their lives, as well as to indicate the inevitable arrival of an event into their lives. Therefore, in this study I also explain both conceptualizations, which Boroditsky (2000) established as two modalities of the same event-related metaphor.

KEYWORDS: metaphoric reasoning; blending; aging; time-space; life path

Fecha de recepción del artículo: 14 de noviembre de 2016

Fecha de recepción de la versión revisada: 15 de junio de 2017

Fecha de aceptación: 20 de junio de 2017

La correspondencia relacionada con este artículo debe dirigirse a:

Alexandra Astrid Ruiz Surget

a.ruiz.surget@gmail.com

Estudios de Lingüística Aplicada, año 35, número 66, diciembre de 2017, pp. 173-203

doi: 10.22201/enallt.01852647p.2017.66.836 


\section{INTRODUCCIÓN}

La idea de este estudio surge a partir de la observación de un fenómeno social ${ }^{1}$ que, si bien en México es incipiente en comparación con Europa, modifica desde hoy las estructuras sociales y los conceptos con los cuales las personas viven cotidianamente: México sigue la tendencia del envejecimiento demográfico que se observa en muchos países desarrollados y en vías de desarrollo.

Sostengo que el aumento en la esperanza de vida, que es un hecho de la realidad, tiene un impacto en la comprensión y creación de otras realidades simbólicas con las que las personas construyen y proyectan su vida cotidianamente. Por lo anterior, dado que el lenguaje es una de las herramientas humanas más poderosas para construir, comprender e interactuar dentro de una sociedad, considero que los recursos lingüísticos de los que se vale una persona para expresar el transcurso de su vida han de cambiar conforme se amplía la expectativa de vida para los miembros de una sociedad.

El objetivo de este estudio es explicar, mediante el modelo teórico de la integración conceptual (Fauconnier \& Turner, 2002), la conceptualización del tiempo en términos de espacio que los sujetos entrevistados construyen en su discurso durante la interacción en una entrevista sobre su trayecto de vida. Los objetivos específicos son los siguientes:

- Establecer los rasgos semánticos compatibles de tiempo y espacio que posibilitan la construcción conceptual del tiempo de vida como un camino.

- Identificar los rasgos semánticos del verbo llegar (utilizado en la forma ir a + infinitivo y en la forma finita) que permiten integrar conceptualmente

1 No deseo caracterizar el fenómeno del envejecimiento demográfico como "el problema" a partir del cual surge mi estudio, ya que, en primer lugar, no deseo problematizar la vejez y, en segundo lugar, no se trata de una situación simple o concreta que se pueda presentar en un artículo, ponencia o discusión para posteriormente desmenuzar y ensayar hipótesis al respecto. Si bien mi estudio se centra en el uso del verbo llegar para referirse a etapas y momentos de la vida, en una escala más grande dicho objeto de estudio lingüístico se inserta en una dinámica social compleja, derivada de la interacción de múltiples actores que tienen sus propias formas de caracterizar la vejez y el paso del tiempo (las instituciones, los centros de salud, la familia, los gerontólogos, las personas de la tercera edad, etc.). Un problema implica una resolución o una respuesta, este estudio busca ayudar a desentrañar la creación de significado en una interacción.

Estudios de Lingüística Aplicada, año 35, número 66, diciembre de 2017, pp. 173-203 doi: 10.22201/enallt.01852647p.2017.66.836 
los dominios de tiempo y espacio para producir metáforas del tipo LA VIDA ES UN CAMINO.

- Mostrar que las metáforas aquí presentadas se comportan conforme a las diferencias entre dos subesquemas de la metáfora TIEMPO ES ESPACIO (el subesquema ego-moving y el subesquema time-moving) propuesto por Boroditsky (2000).

Me detengo ahora en algunos datos estadísticos para proporcionar un contexto acerca del fenómeno de envejecimiento demográfico al cual me he referido al inicio. El envejecimiento demográfico es el "incremento de la población con 60 o 65 años de edad dependiendo del nivel de desarrollo del país; este crecimiento es una consecuencia del descenso de la mortalidad y fecundidad a partir de las primeras etapas identificadas como transición demográfica" (Montes de Oca, 2010: 162).

En 2010, el Consejo Nacional de Población (Conapo) contabilizó 5064979 personas mayores de 65 años en todo el país y calculó 8256291 en 2015. Se estima que para 2030 sean 14089232 y para 2050 la cifra podría llegar a 24362839 personas, por lo que esta franja de edad habrá triplicado su tamaño en tan solo 35 años a partir de 2015. En cuanto a la esperanza de vida: en 2015 fue de 74.9 años a nivel nacional (72.3 para los hombres y 77.7 para las mujeres). Se estima que en 2030 la esperanza de vida al nacer sea de 74.6 años para los hombres y 79.4 para las mujeres. Esto significa que en el lapso de un siglo, habremos podido aumentar más del doble la duración de la vida de los mexicanos.

La vejez se ha convertido en un fenómeno colectivo pues es una etapa más de la vida a la que la mayoría de los miembros de una sociedad tendrá acceso. Cumplir 70 años no es ya un caso excepcional sino la norma en una comunidad,

Lo anterior necesariamente modifica la composición social y la propia conceptualización de lo que es "ser viejo". Si en la década de 1930 la esperanza de vida de un mexicano apenas superaba los 30 años de edad, el concepto de vejez seguramente era muy diferente al que tenemos hoy. De igual manera, una esperanza de vida de tres décadas en promedio establecía parámetros muy distintos para evaluar hitos de vida, tales como el matrimonio, el trabajo o la reproducción. Hoy en la Ciudad de México, un joven de 25 años que esté terminando sus estudios y aún viva con sus padres se evalúa como "normal" en ciertos grupos 
sociales; la edad para casarse o tener el primer hijo también se ha ido "recorriendo" o "atrasando" con respecto a décadas anteriores.

La vejez reconfigura la dinámica social, por lo tanto, las prácticas lingüísticas en torno a dicho fenómeno también se modifican para describirlo e integrarlo a la vida cotidiana. Si la ciencia nos promete setenta años de existencia, las posibilidades para realizar nuestra vida se multiplican, así como las metáforas para imaginar nuestros proyectos y etapas de vida.

\section{METÁFORAS DE TIEMPO-ESPACIO}

En este estudio abordo la metáfora desde su definición como herramienta cognitiva para construir una realidad (Lakoff \& Johnson, 1986 [1980]; Bergen, 2012), ya que si bien la metáfora emerge a la superficie lingüística en el discurso, es a la vez un poderoso mecanismo imaginativo de integración de conceptos. La construcción de una realidad gracias a un proceso metafórico de pensamiento - que, por supuesto no es el único- se da en la interacción constante del individuo con los demás miembros de su comunidad. Tenemos entonces tres aristas desde las cuales se debe considerar un fenómeno lingüístico, como puede ser el de la metáfora, según Van Dijk (1999, 2001): la observación de un fenómeno que impacta las estructuras sociales humanas, la descripción del fenómeno lingüístico que da cuenta de ese cambio social y la interfaz cognitiva que permite la construcción de significado acerca de ese fenómeno.

De acuerdo con lo anterior, defino la metáfora como sigue:

- Desde el eje cognitivo, la metáfora es una forma de razonamiento mediante la cual el ser humano construye sentido al integrar dos o más dominios conceptuales (Fauconnier \& Turner, 2002).

- Desde el eje linguiístico-discursivo, el razonamiento metafórico se manifiesta en el discurso como una instanciación lingüística cuya construcción semántica emerge en la interacción.

- Desde el eje social, la metáfora cumple la función social de actualizador de marcos conceptuales dado que nuevos significados emergen de la integración conceptual y permiten al individuo comprender y compartir una construcción de la realidad (Ruiz, 2011). 
En su ya clásico libro Metaphors we live by, Lakoff y Johnson indican que la metáfora "impregna la vida cotidiana, no solamente el lenguaje, sino también el pensamiento y la acción. Nuestro sistema conceptual ordinario, en términos del cual pensamos y actuamos, es fundamentalmente de naturaleza metafórica" (1986 [1980]: 39). Las teorías conceptuales de la semántica cognitiva, como la de Lakoff y Johnson y después otras (Fauconnier, 1997; Kövecses, 2002; Wilson \& Gibbs, 2007), se han encargado de dejar claro que el razonamiento metafórico se encuentra entre las capacidades cognitivas más importantes, productivas y cotidianas del cerebro humano.

En cuanto al concepto de tiempo, los estudios que explican su conceptualización en términos de espacio demuestran que esta conceptualización es metafórica (Boroditsky, 2000; Núñez \& Cooperrider, 2013; Wittmann, 2013). Conceptualizamos las sensaciones de que ocurren cambios en nuestro entorno y en nuestro cuerpo a través de modelos mentales en donde un suceso se encuentra adelante o atrás de nosotros; también podemos acudir a un modelo mental en el que nosotros somos quienes nos movemos hacia el suceso. Estas representaciones se dan naturalmente a través de las diferentes culturas y con sus respectivas variantes presentes en las lenguas. Por ejemplo, el inglés y el mandarín muestran una representación del futuro que se encuentra frente a una persona y del pasado, detrás; asimismo, el mandarín también emplea un eje de verticalidad para referirse al tiempo en donde el pasado se encuentra arriba y el futuro, abajo (Boroditsky, 2000). ${ }^{2}$

La metáfora ontológica ${ }^{3}$ TIEMPO ES ESPACIO es la metáfora general de la cual parto para analizar las metáforas producidas en las narrativas de las perso-

2 Véase también el estudio de Núñez, Neumann y Mamani (1997) acerca de la conceptualización del tiempo en aymará y Fedden y Boroditsky (2012) sobre el hebreo, el mian y el chino y la influencia de la escritura de derecha a izquierda en la conceptualización del tiempo.

3 Lakoff y Johnson (1986 [1980]) definen la metáfora ontológica como una metáfora que se construye a partir de nuestra experiencia corporal con objetos y sustancias del mundo, por ejemplo: recipientes, líquidos, entidades discretas, fuerzas físicas, los sentidos de la percepción, etc. De esta manera, un sentimiento como el amor o el odio se puede conceptualizar en términos de un objeto cuantificable: mucho, un poco, tanto, demasiado, etc. La vida se puede conceptualizar en términos de un camino, lo cual le confiere estructura, límites espacio-temporales y puntos de referencia. 
nas entrevistadas en este estudio. Filósofos, poetas, científicos y, más recientemente, lingüistas han estudiado el tiempo en términos de espacio. Como expresan Núñez y Cooperrider:

These and other time concepts, such as duration and sequence, belong to the realm of high-level cognition: as observed in humans, they are mediated by language and culture, but are also firmly rooted in bodily experience and realized by neural mechanisms that are as yet poorly understood. Everyday time concepts presumably developed as an efficient way of understanding the rich - and yet fundamentally abstract - organization of temporal experience and of coordinating these understanding with others (2013: 220). ${ }^{4}$

En el discurso que busca relatar episodios de vida y ejemplificar proyectos y facetas identitarias, los individuos emplean un subtipo mucho más concreto de metáfora en donde la vida es un camino. Esta metáfora presenta límites más claros y asequibles para el pensamiento humano. Por ejemplo, en vez de tiempo, tenemos el concepto vida, el cual nos indica un lapso de alrededor de 70 años y ya no un tiempo indefinido que podría ser infinito. Cada persona conceptualiza el tiempo de su vida de acuerdo con una asequible escala de los años que ha vivido y espera vivir. Lo mismo ocurre con el concepto general de espacio, el cual adquiere referentes más concretos cuando lo trasladamos a la estructura de un camino: tiene un punto de partida y un destino, así como un recorrido. Permite, además, construir un significado de la vida como un trayecto, en el que se pueden hacer pausas, tomar atajos, adelantar etapas o llegar tarde a otras. Esto dota de infinitas posibilidades conceptuales al sentido de vivir.

4 "Estos y otros conceptos, como la duración y la secuencia, pertenecen al dominio de la alta cognición: tal como se observa en los seres humanos, dichos conceptos están mediados por el lenguaje y la cultura, pero también están firmemente enraizados en la experiencia corporal y ejecutados por mecanismos neuronales que apenas comienzan a comprenderse. Los conceptos cotidianos temporales posiblemente se desarrollaron como una forma eficaz para entender la rica - y fundamentalmente abstracta - organización de la experiencia temporal y para coordinar esta comprensión con otras" (traducción nuestra). 


\section{METÁforas DE TIEMPO-ESPACIO CON EL VERBO LLEGAR}

En este artículo me enfoco en las metáforas que provienen del esquema LA VIDA ES UN CAMINO, instanciado en metáforas con el verbo de movimiento llegar.

Elegí analizar las metáforas regidas por el verbo llegar, ya que fue uno de los verbos más utilizados por todos los individuos para referirse al trayecto por las diferentes etapas de su vida. En las casi 17 horas de grabación recabadas a partir de todas las entrevistas, el verbo llegar para referirse a cambios en la vida de la persona emergió reiteradamente, logrando estructurar de manera importante la conceptualización del tiempo en términos de espacio. Los siguientes son fragmentos más amplios de discurso que ejemplifican el uso al que me refiero como objeto de análisis de este artículo:

(1) No pues, ya 90, pues ya pasó la línea casi casi, o sea 90 es: felicidades, ¿cómo llegaste ahí? [Jimena, 22 años, acerca de cumplir 90 años]

(2) Pero... ¿y sabes qué? Y esa es la presión que tenemos nosotros ahorita. De decir es que hay que hacer dinero, es que es ahorita y si no... Porque si llegas a los 30 y no has hecho esto, ya valió. Y luego estás en los treinta y dices si llego a los 40... [Rodrigo, 33 años]

(3) Si llegas a esta etapa de los 40 ya tienes un reto de... si en esas primeras etapas no te fue, vamos a decir que pues a lo mejor estudiaste lo que no era o los trabajos no te gustaron o realmente no has logrado cubrir ciertas metas o alcanzar a conocer mejor las cosas realmente los 40 se empieza ya a dificultar, porque las oportunidades ya están para gente que ya te lleva ventaja entonces si eres los de ventaja como va en mi caso, pues muy bien porque estás en las grandes ligas y hay oportunidades padrísimas y divertidas que, pues a lo mejor fueron un sueño medio guajiro cuando tenías 20 y te graduaste y, vamos: hoy es una posibilidad. [Lorena, 46 años]

El uso del verbo llegar manifiesta un modelo mental en el que el individuo se desplaza por el camino de su vida hacia las diferentes edades, mismas que describe o evalúa según su experiencia y parámetros sociales. Propongo aquí, a través del modelo de integración conceptual, los rasgos semánticos compartidos 
por tiempo y espacio. Esto permitirá observar qué rasgos del verbo llegar son compatibles con tiempo.

Para entender la interacción conceptual que propongo, es necesario explicar los rasgos semánticos de llegar. El verbo llegar codifica el desplazamiento en relación con una dirección, como también lo hacen subir, volver o venir, por ejemplo. Se trata de un verbo que focaliza el momento puntual de la terminación del evento pues perfila el final de la trayectoria de un objeto o persona. Con llegar se puede expresar la culminación de la acción de cambiar de un lugar a otro. En ese sentido, Vendler (1957) lo caracteriza como logro: es + dinámico, - durativo y + télico. De acuerdo con Arellanes (2005), el verbo llegar corresponde a un verbo de evento instantáneo. Los verbos de evento instantáneo generalmente dan lugar a un estado resultante. En el caso de llegar, el resultado es la presencia de algo o alguien, que previamente no estaba, en un lugar, por lo que se clasifica dentro del subgrupo de verbos locativos de evento instantáneo (al igual que irse, aterrizar, entrar, alcanzar).

Para este análisis interesan las metáforas obtenidas durante la interacción con los entrevistados en donde el verbo llegar se utiliza para indicar un cambio de edad o etapa:

(4) a. Entonces en algún momento llegaremos [a la vejez]. [Yolanda, 22 años]

b. Yo creo el más grande [de los colegas] no llega a los 50. [Cristian, 36 años]

c. Y qué pasa cuando ya perdiste la identidad tú, individual, en cuanto llegas a pareja. Luego llegas a hijos, otro cacho. [Magda, 34 años]

Ahora bien, las metáforas identificadas estaban principalmente formadas con la perífrasis ir a infinitivo. Por lo tanto, los rasgos semánticos anteriormente mencionados no son los únicos que deben considerarse para la integración conceptual. Genta indica que las perífrasis con infinitivo sugieren una potencialidad futura: "las perífrasis verbales de infinitivo producen un valor potencial de futuro (no es un futuro temporal). Es el valor que la tensión temporal en potencia (no realizada) proyecta" (2008: 165).

En cuanto a la perífrasis específica ir a +infinitivo, Bravo establece que hay una diferencia fundamental entre dicha perífrasis y el futuro simple (voy a cantar/cantaré): se trata de una "situación futura como conectada con un estado de 
cosas previo" (2008: 11). La autora concuerda con García Fernández (2000) en que la perífrasis tiene un aspecto prospectivo: es +intencional y +inevitable. Es una acción única y no habitual, en contraposición con el posible aspecto demarcativo cuando enfatiza un evento habitual. Bravo ofrece estos ejemplos para explicar la diferencia entre la perífrasis demarcativa (5) y la prospectiva (6):

(5) Cada vez que mis vecinos tiraban un petardo, el petardo iba a explotar al lado de mi ventana.

(6) El petardo iba a explotar al lado de mi ventana, pero lo apagaron a tiempo (Bravo, 2008: 54).

En el caso de las metáforas con el verbo llegar, opino que se trata de un uso prospectivo por estar en estrecha relación con el evento previo y por ser un momento puntual, único, en la vida de la persona. Tal es el caso de los ejemplos que se muestran en $(7 \mathrm{a}-\mathrm{c})$ :

(7) a. Yo voy a llegar a esa edad en algún momento de mi vida. [Yolanda, 23 años]

b. Sí voy a llegar bien [a la vejez], aunque sea con la cabeza blanca. [Marco, 40 años]

c. Es como el juego de serpientes y escaleras: yo ya iba a llegar a la meta y de repente me regresaron a las piñatas y al kínder. ${ }^{5}$ [Jimena, 22 años]

Esta somera caracterización del verbo llegar y su perífrasis con ir a conforma el contenido de uno de los espacios conceptuales que propongo en la aplicación

5 Bravo (2009: 292) sostiene que la perífrasis en pretérito imperfectivo iba a + infinitivo mantiene el aspecto prospectivo de inminencia e intencionalidad de la acción; la diferencia principal con respecto al presente estriba en que establece la inminencia de una acción anclada a una esfera del pasado. Por ejemplo: a. ETA iba a atentar en la estación de Chamartín el día de Nochebuena, pero la policía descubrió a tiempo los planes y pudo impedir la masacre. b. Juan iba a llamarla a las tres (, pero finalmente cambió de opinión). c. La excursión iba a ser mañana (, pero la han suspendido). En el caso de las metáforas con iba a + infinitivo (7c), el sujeto estaba a punto de superar una etapa de vida pero fue "devuelto" al inicio de la misma: se refiere a que a los 45 años, con la hija menor ya casi en la prepa y la mayor saliendo de la universidad, el individuo se sentía cerca de culminar la etapa de estar al pendiente de los estudios y de los pagos de la educación de las hijas; y entonces fue padre por cuarta vez.

Estudios de Lingüística Aplicada, año 35, número 66, diciembre de 2017, pp. 173-203

doi: 10.22201/enallt.01852647p.2017.66.836 
del modelo de la integración conceptual. Pero antes de pasar a ello, me detengo en la metodología con la que fueron obtenidas las narrativas que contienen las metáforas que analizo en este artículo.

\section{Metodología}

Los ejemplos que analizo en este artículo forman parte de un corpus mucho mayor de metáforas recabadas a partir de las narrativas de las 14 personas entrevistadas en el año 2016 para mi proyecto de investigación doctoral. Para el estudio que realicé, me enfoqué en los casos de habitantes de la Ciudad de México, la entidad federativa con más población adulta mayor de 65 años $(7.9 \%$, en comparación con el promedio nacional de $7.2 \%$ ) y con mayor índice de envejecimiento (33\%, en comparación con el promedio nacional de 21\%) (INEGI, 2001).

Dicho fenómeno demográfico implica desde ahora un cambio en la dinámica social y en la conformación de nuevos actores sociales, por lo que entrevisté jóvenes profesionistas que, con la perspectiva del "resto de su vida por delante", plantearon sus proyectos familiares y profesionales en el marco de estas nuevas circunstancias sociales.

La entrevista semi-estructurada resultó ideal como técnica de obtención de datos para investigar la conceptualización del espacio-tiempo ontológico de la vida, ya que permite un espacio de co-construcción de significado. Si bien se trata de una interacción, quien expuso la conceptualización de su vida fue el sujeto entrevistado.

Los entrevistados cuentan con los recursos materiales y culturales suficientes para cubrir sus necesidades básicas. Asimismo, cuentan con recursos adicionales que generan la necesidad de diseñar estrategias y planes encaminados al aprovechamiento de esos recursos y, especialmente, al crecimiento personal y a la búsqueda de la felicidad. Sin embargo, se encuentran constreñidos, dado que esos recursos adicionales no son suficientes para realizar todos los proyectos que quisieran. Pertenecen a un nivel socio-económico $\mathrm{C}+(\mathrm{NSE} / \mathrm{C}+) .{ }^{6} \mathrm{De}$ acuerdo a su edad, quedaron agrupados de esta forma:

6 Se refiere al nivel socioeconómico según la clasificación de la Asociación Mexicana de Agencias de Investigación (AMAI). La asociación calcula, mediante un algoritmo que incluye factores 
Grupo 1: Dos hombres y dos mujeres en la segunda década de vida.

Grupo 2: Cuatro mujeres y dos hombres en la tercera década de su vida.

Grupo 3: Dos hombres y dos mujeres en la cuarta década de su vida.

El grupo 1, por las características del NSE, tiene un perfil más homogéneo: se trata de estudiantes universitarios próximos a titularse que se encuentran en la etapa de inserción laboral. En los grupos 2 y 3 se tienen ya diferentes perfiles, derivados de las decisiones de vida que ya han podido tomar: empleados, emprendedores y profesionistas por cuenta propia, con pareja estable o solteros, con y sin hijos. En la entrevista se tocaron los siguientes ejes temáticos:

- Conceptualización de la vejez: el entrevistado comparte la imagen y los proyectos que tiene para su vejez. También se explora el concepto de vejez que tiene la persona.

- Actividad actual: este tema busca que el entrevistado se posicione sobre el espacio-tiempo de su vida en cuanto al ámbito profesional. Emite juicios de valor (prematuro, a tiempo, tardío) con respecto de su propia trayectoria y la de otros.

- Retrospectiva laboral: salvo por el grupo 1, el resto de los grupos construye un relato de sus primeras experiencias profesionales y les confiere un significado a la luz del posicionamiento realizado en el marco conceptual que corresponde a su situación actual. Es decir, el sentido de la trayectoria profesional se evalúa con elementos tanto del presente como del pasado.

- Estrategias de auto-gestión y optimización: se hacen planteamientos directos sobre cómo llevan a cabo estrategias de cuidado y auto-gestión de su cuerpo y su salud.

- Plan de vida: el entrevistado comunica qué desea conseguir en el largo plazo en los planos laboral, patrimonial, amoroso y familiar.

de bienestar económico, material y cultural, el nivel socioeconómico de una persona, y potencial consumidora de servicios. En el caso del NSE C/C+, las personas cuentan con recursos para cubrir sus necesidades básicas y adicionalmente tienen recursos que pueden destinar a la educación, viajes, inversiones y planeación del patrimonio. Véase www.amai.org (Consulta: 25 de junio, 2015). 


\section{LA METÁFORA DESDE LA INTEGRACIÓN CONCEPTUAL}

El modelo de la integración conceptual ha ayudado a explicar por qué cuando estamos ante una metáfora encontramos en su significado algunos rasgos que no parecen provenir de los significados originales de sus constituyentes. La expresión (o instanciación) lingüística que proviene de los rasgos de los inputs - o espacios de ingreso- es novedosa y creativa.

En el modelo básico de la integración conceptual (Fauconnier, 1984, 1997; Fauconnier \& Turner, 2002) se plantean dos espacios mentales llamados inputs o espacios de ingreso, en vista de que en ese espacio ingresan representaciones mentales que son producto de la experiencia y la cultura. Dichos espacios de ingreso pueden ser modificados y manipulados para establecer relaciones con otras estructuras a través de diferentes operaciones mentales (Fauconnier, 1984: 32).

Un tercer espacio mental, el espacio genérico, se llama así porque en él se construye una estructura normalmente muy básica que resalta características relevantes y los rasgos coincidentes entre las estructuras de los dos inputs. El resultado de la integración conceptual que da origen a la instanciación se llama blend. Dicha estructura emergente es producto del encuentro e interacción de dos dominios cognitivos o inputs. Gracias a la propiedad emergente del blend, el lenguaje puede producir instanciaciones como la metáfora. Siguiendo a los autores, cuando ocurre una integración conceptual o blending sucede lo siguiente (Fauconnier, 1997: 149-150):

1) Cross-space mapping: There is a partial mapping of counterparts between the input spaces $I_{1}$ and $I_{2},[\ldots]$.

2) Generic space: There is a generic space, which maps onto each of the inputs. This generic space reflects some common, usually more abstract structure and organization shared by the inputs and defines the core cross-space mapping between them $[\ldots]$.

3) Blend: The input $I_{1}$ and $I_{2}$, are partially projected onto a fourth space, the blend. 
4) Emergent structure: The blend has emergent structure not provided by the inputs. $^{7}$

Lo anterior se ilustra en la Figura 1.

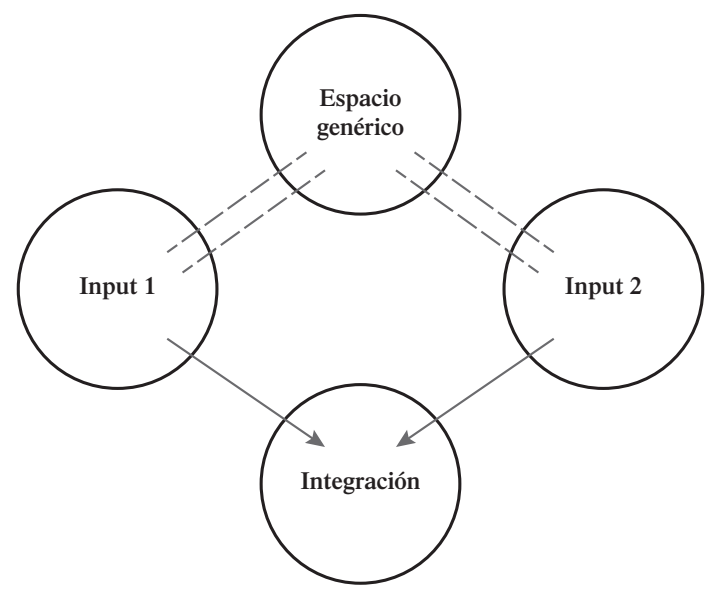

FiguRA 1. Modelo de la integración conceptual (Fauconnier \& Turner, 2002)

En el modelo mostrado en la Figura 1, las líneas punteadas que unen el espacio genérico con los input 1 y 2 buscan ilustrar las estructuras y elementos que tanto input 1 como input 2 tienen en común. Dichos rasgos comunes son los que conforman el espacio genérico. Las flechas que descienden desde input 1 y 2 ilustran los elementos que finalmente se manifiestan — lingüísticamente en ese caso- en

7 "1. Mapeo entre espacios de ingreso: se da un mapeo parcial de contrapartes entre los espacios de ingreso $I_{1}$ e $I_{2},[\ldots]$.

2. Espacio genérico: hay un espacio genérico, que se mapea sobre cada espacio de ingreso. Este espacio genérico refleja una estructura y organización comunes, usualmente más abstractas, a los espacios de ingreso y define el núcleo del mapeo entre dichos espacios de ingreso $[\ldots]$.

3. Integración: los espacios de ingreso, $I_{1}$ e $I_{2}$, se proyectan parcialmente hacia un cuarto espacio, la integración conceptual.

4. Estructura emergente: la integración tiene una estructura emergente, que no ha sido proporcionada por los espacios de ingreso" (traducción nuestra). 
la interacción social, la charla. La integración es la estructura que emerge a partir de la interacción de input 1 y 2.

\subsection{Integración conceptual de las metáforas con el verbo llegar}

Para ejemplificar el funcionamiento de la integración conceptual, será útil abordar un primer caso, extraído del ejemplo (3) del discurso de Lorena que presenté antes: Si llegas a esta etapa de los 40 ya tienes un reto. En este ejemplo tenemos un espacio de ingreso o input 1 que corresponde a espacio y un input 2 que corresponde a tiempo.

En cuanto a los rasgos conceptuales del input 1 (espacio) que ingresan a partir de un marco conceptual más preciso como el movimiento lexicalizado en el verbo llegar, los rasgos funcionales para esta integración conceptual son:

- Movimiento: es posible moverse de múltiples maneras y en varias direcciones en un espacio. Dicho movimiento resulta en un cambio de lugar.

- Cambio de lugar: resultado de verbos de desplazamiento con direccionalidad, como llegar.

- Trayectoria o dirección: si bien hay maneras de moverse, llegar codifica trayectoria, por lo que manera no será una propiedad emergente del blend.

- Origen: un espacio o lugar puede ser el origen del desplazamiento. En la expresión de Lorena no emergió este origen aunque sí forma parte del contenido conceptual del input 1.

- Destino: un espacio o lugar puede ser el destino del desplazamiento. En la expresión de Lorena sí emerge este rasgo del input 1.

- Entidad que se mueve: Langacker (1987) la denomina trajector. Se trata de la persona u objeto que realiza el desplazamiento. En este caso, Lorena utiliza el tú impersonal para explicar su trayectoria de vida y elaborar algunas generalidades que caracterizan a la gente de su edad. ${ }^{8}$

8 Se trata, como lo describe Hidalgo Navarro (1997) de una "estrategia pragmalingüística" que se puede orientar a la generalización, a la modestia o a alguna otra interacción en la esfera comunicativa. Pienso que los sujetos de mi investigación, que la emplean frecuentemente, buscan presentar aspectos generales de las etapas de vida que describen utilizando esta estrategia; no es 
En cuanto al input 2, que contiene los rasgos de tiempo, el tiempo es un concepto tan abstracto que solo puede pensarse o notarse en los cambios de estado (de posición, de color, de aspecto, etc.) de las cosas. Por lo tanto, dentro del input 2, propongo:

- Cambios de estado: esta experiencia cotidiana y corporeizada nos compele a explicar dichos cambios y medirlos utilizando la terminología que hemos inventado para el tiempo.

En el espacio genérico se resaltan relaciones vitales ${ }^{9}$ de similitud entre los espacios 1 y 2, las cuales deben ser compatibles entre sí para ingresar al espacio genérico; en este caso, cambio de lugar y cambio de estado. El cambio de estado, ligado al paso del tiempo, puede verse en un objeto que primero está ausente de un lugar y luego está presente. La relación básica que se puede colocar en el espacio genérico es cambio.

La integración conceptual o blend es la expresión linguiística que emerge de esta metáfora (Figura 2). En el ejemplo (3), la persona (Lorena o en general quien llegue a los 40) llega a un "lugar" en el que previamente no estaba, es decir, el punto del camino vital que corresponde a los 40 años.

La clave de la metáfora (Figura 2) se encuentra en la posibilidad de equiparar momentos de vida con lugares. Como indiqué anteriormente, la metáfora ontológica TIEMPO ES ESPACIO, cuya versión conceptual más concreta y delimitada es LA VIDA ES UN CAMINO permite hablar de una edad como si fuera un lugar. Gracias al esquema mental presente en LA VIDA ES UN CAMINO, Lorena ha podido hablar de un cambio de edad como si se tratara de un desplazamiento hacia un siguiente punto del camino de su vida.

aquí el objeto principal de análisis, solo añadiré que quizá sea una estrategia que busca incluirme o acercarme, al menos, a sus experiencias.

9 Fauconnier y Turner (2002) se refieren a relaciones vitales para caracterizar una serie de relaciones lógicas entre los conceptos contenidos en los espacios mentales. Por ejemplo, las relaciones de identidad, tiempo, espacio y causa-efecto, por mencionar algunas. Dichas relaciones revelan una comprensión del mundo que es el objetivo primordial de la operación mental llamada integración conceptual. 


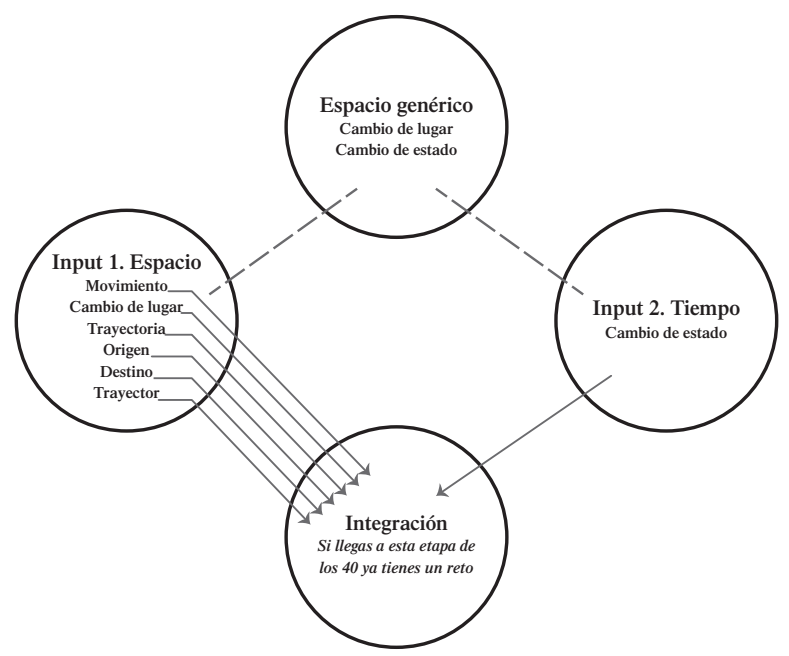

FIGURA 2. Esquema de la integración conceptual de la metáfora tiempo-espacio regida por el verbo llegar mostrada en el ejemplo (3)

Cabe observar que en la Figura 2, que ilustra el ejemplo de Lorena, no ocurre un traslado "simétrico" de rasgos hacia el espacio genérico: input 1, "contiene" muchos rasgos más que el input 2 pero tiene en común la relación cambio, por lo que solo ese rasgo se ha graficado como el que "sube" al espacio genérico. De igual manera, al momento de la enunciación de la metáfora, el contenido casi completo del input 1 viene a darle estructura a la construcción del ejemplo (3). Aquí pueden encontrarse nuevamente los rasgos del input 1, excepto origen, que no emergió:

- Movimiento por una dimensión espacial, que en el caso de llegar es un desplazamiento con dirección.

- Destino, es decir, los 40.

- Cambio de un lugar (edad previa) a otro.

- Trayectoria, es decir, la vida.

- Trayector: Lorena o cualquier individuo en su situación.

El rasgo único del input 2 está presente también en la instanciación: el cambio que produce el paso del tiempo en la vida de Lorena. 
Esta integración conceptual corresponde a los ejemplos de metáforas (1-4) y (7), en donde una persona llega a una etapa de vida. En unos casos, los sujetos entrevistados hablan de terceros, de sí mismos o generalizan con el pronombre de segunda persona pero en todos los casos se trata del mismo tipo de trayector: + humano, + volitivo y + activo.

Con respecto a quién ejecuta el desplazamiento, hay una variante importante en muchos otros ejemplos de metáforas que emergieron en el discurso durante las entrevistas. En $(8 \mathrm{a}-\mathrm{c})$ se observa que dicha entidad que ejecuta el movimiento es un momento.

(8) a. Va llegar un momento en que esos descuidos van a caer en un shock. [Magda, 34 años]

b. Va a llegar un cierto momento en la vida en que yo quiera bajarle al estrés. [Marco, 40 años]

c. Va llegar un momento en que no me va encantar estar ahí. [Marco, 40 años]

En estos ejemplos $(8 \mathrm{a}-\mathrm{c})$ no se trata de una persona sino de un momento o punto que "llega". Para estos casos, propongo que los rasgos del input 1 que emergen en los blends sean los siguientes:

- Rasgo del movimiento, caracterizado por un desplazamiento.

- Cambio de lugar al sitio donde se encuentra el sujeto.

- Destino, que es el sitio de la interacción entre los sujetos (entrevistado-investigadora).

- Trayector que no es un agente. No es humano ni tiene volición ni masa. Su dimensión física en realidad no es observable ni palpable. Este trayector proviene del dominio del input 2: es el tiempo.

Un momento, que pertenece al dominio conceptual de tiempo y que no es un objeto concreto, llega. Esta importante diferencia se observa en la Figura 3, que ilustra el ejemplo (8b).

Boroditsky (2000) propone dos esquemas de la metáfora espacio-temporal que resaltan trayectores diferentes al momento de conceptualizar el tiempo: ego-moving metaphor y time-moving metaphor. En el primero, el observa- 


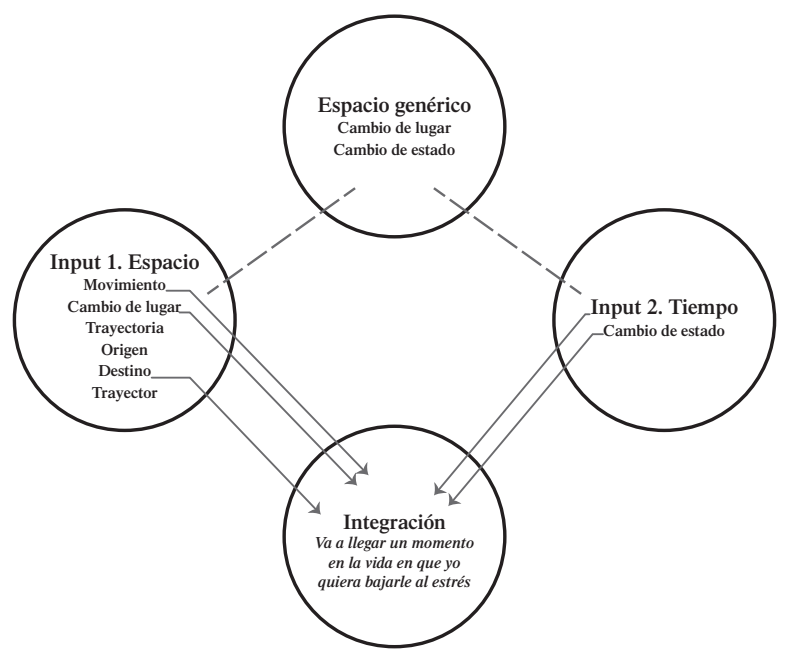

FIGURA 3. Esquema de la integración conceptual de la metáfora tiempo-espacio regida por el verbo llegar con foco en el momento como fuerza mostrada en el ejemplo (8b)

dor-conceptualizador se mueve hacia el futuro (ejemplos (1-4) y (7)) y, en el segundo, el tiempo se mueve en una especie de flujo o río hacia el conceptualizador (ejemplo (8)). Se trata entonces, de acuerdo con la autora, de dos subesquemas de la metáfora espacio-temporal generados por un cambio de foco: ya sea hacia el agente que avanza por la línea del tiempo o hacia el tiempo que transita hacia el individuo.

En el subesquema time-moving, el momento o punto del tiempo se convierte en la entidad más prominente (trajector) y el sujeto se convierte en el punto de referencia (landmark) del esquema. Por lo tanto, los rasgos trayectoria y origen ya no emergen en dicha metáfora (Figura 3). Las propiedades - humano y + abstracto del momento no permiten conceptualizarlos por un camino como en el que sí podemos imaginar a Lorena (ejemplo (8)). Si bien en los ejemplos (1-4) y (7) del subesquema ego-moving el rasgo origen tampoco emerge, sí se puede inferir o imaginar dentro de la lógica del subesquema y del esquema general de LA VIDA COMO UN CAMINO: el origen del trayecto es el nacimiento y el destino final es la muerte. Así, el ejemplo (8) se produce a partir de la conceptualización time-moving metaphor en donde la línea de tiempo se desliza hacia el individuo. 


\subsection{Rasgos que permiten la conceptualización del tiempo a través de metáforas con el verbo llegar}

En esta sección explico por qué el verbo llegar, tanto en su forma finita como en perífrasis, resulta adecuado para integrar los conceptos de tiempo y espacio a través de su semántica.

En los Cuadros 1, 2 y 3 se han ordenado ejemplos de metáforas, extraídas del corpus de las entrevistas, como sigue: cada cuadro está dividido por sujetos (4 sujetos entrevistados en los grupos 1 y 3 , y 6 sujetos entrevistados en el grupo 2); además, las metáforas se han agrupado de acuerdo con el foco que presentan (ego-moving/time-moving), y, finalmente, se han subdividido de acuerdo con la forma que presenta el verbo llegar (en perífrasis o en forma finita).

Cuadro 1. Ejemplos de metáforas con el verbo llegar del grupo 1 (20-30 años)

\begin{tabular}{|c|c|c|c|}
\hline Sujeto & FORMA & EGO-MOVING & TIME-MOVING \\
\hline \multirow[t]{3}{*}{ Yolanda } & ir a + infinitivo & $\begin{array}{l}\text { Yo voy a llegar a esa edad en algún momento } \\
\text { de mi vida }\end{array}$ & $\emptyset$ \\
\hline & & Porque vas a llegar a eso & $\emptyset$ \\
\hline & Finita & $\begin{array}{l}\text { Entonces en algún momento llegaremos [a } \\
\text { la vejez] }\end{array}$ & $\emptyset$ \\
\hline \multirow[t]{2}{*}{ Raúl } & ir a + infinitivo & $\emptyset$ & $\begin{array}{l}\text { Como que siempre sentía que iba a } \\
\text { llegar el momento }\end{array}$ \\
\hline & Finita & $\emptyset$ & $\begin{array}{l}\text { Siempre llega un punto en el que el } \\
\text { cuerpo ya no aguanta }\end{array}$ \\
\hline \multirow[t]{2}{*}{ Jimena } & ir a + infinitivo & $\begin{array}{l}\text { Es como el juego de serpientes y escaleras: } \\
\text { yo ya iba a llegar a la meta y de repente me } \\
\text { regresaron a las piñatas y al kínder }\end{array}$ & $\emptyset$ \\
\hline & Finita & 0 sea 90 es felicidades. ¿Cómo llegaste ahí? & $\emptyset$ \\
\hline \multirow[t]{2}{*}{ Diego } & ir a + infinitivo & $\emptyset$ & $\emptyset$ \\
\hline & Finita & Pues hasta ahí llegaste [60 años] & $\emptyset$ \\
\hline
\end{tabular}

Estudios de Lingüística Aplicada, año 35, número 66, diciembre de 2017, pp. 173-203 doi: 10.22201/enallt.01852647p.2017.66.836 
CuADRo 2. Ejemplos de metáforas con el verbo llegar del grupo 2 (30-40 años)

\begin{tabular}{|c|c|c|c|}
\hline Sujeto & Forma & EGO-MOVING & TIME-MOVING \\
\hline \multirow[t]{2}{*}{ Cristian } & ir a + infinitivo & $\emptyset$ & $\emptyset$ \\
\hline & Finita & Yo creo el más grande no llega a los 50 & $\emptyset$ \\
\hline \multirow[t]{2}{*}{ Lucy } & ir a + infinitivo & $\emptyset$ & $\begin{array}{l}\text { No sé si es porque pensaron que este } \\
\text { momento no iba a llegar }\end{array}$ \\
\hline & Finita & $\emptyset$ & $\emptyset$ \\
\hline \multirow[t]{4}{*}{ Rodrigo } & ir a + infinitivo & $\emptyset$ & $\emptyset$ \\
\hline & Finita & Si llegas a los 30 y no has hecho esto, ya valió & $\emptyset$ \\
\hline & & $\begin{array}{l}\text { Y luego estás en los treinta y dices si llego a } \\
\text { los } 40 \ldots\end{array}$ & \\
\hline & & $\begin{array}{l}\text { Si tenemos } 10 \text { puntos que queremos hacer, } \\
\text { llegamos al fin del año y logramos conseguir } 3\end{array}$ & \\
\hline \multirow[t]{4}{*}{ Magda } & ir a + infinitivo & $\emptyset$ & $\begin{array}{l}\text { Va a llegar un momento en que esos } \\
\text { descuidos van a caer en un shock. }\end{array}$ \\
\hline & Finita & $\begin{array}{l}\text { De repente te das cuenta que, tienes un sueño } \\
\text { y llegas a cierta edad y chin ya hiciste, caíste } \\
\text { en lo de usos y costumbres más de lo que } \\
\text { quieres }\end{array}$ & $\begin{array}{l}\text { Pero llega un momento en el que dices } \\
\text { mi mamá nunca me ha dicho que no }\end{array}$ \\
\hline & & $\begin{array}{l}\text { Y que pasa cuando ya perdiste la identidad tú, } \\
\text { individual, en cuanto llegas a pareja. Luego } \\
\text { llegas a hijos, otro cacho. }\end{array}$ & $\begin{array}{l}\text { Y de repente llega un momento en que } \\
\text { digo ahorita yo estoy en el trabajo y él } \\
\text { está en su trabajo y tenemos diferentes } \\
\text { experiencias. }\end{array}$ \\
\hline & & En qué momento llegamos a esto ¿no? & \\
\hline Tania & & $\emptyset$ & $\emptyset$ \\
\hline Myriam & ir a + infinitivo & $\emptyset$ & $\emptyset$ \\
\hline & Finita & $\emptyset$ & $\begin{array}{l}\text { Llega un momento en que ya ni puedes } \\
\text { hablar. }\end{array}$ \\
\hline
\end{tabular}


CuADRo 3. Ejemplos de metáforas con el verbo llegar del grupo 3 (40-50 años)

\begin{tabular}{cccc}
\hline Suseto & Forma & EGO-MOVING & TimE-MOVING \\
\hline Flavio & ir a + infinitivo & $\emptyset$ & $\emptyset$ \\
& Finita & $\begin{array}{l}\text { Muy probablemente yo llegue a los } 80 \\
\text { rodeado de un buen grupo de amigos }\end{array}$ & $\emptyset$
\end{tabular}

Lorena ir a + infinitivo

Finita

Finita

Julieta $\emptyset$

Si llegas a esta etapa de los 40 ya tienes un reto

Ya llegas a cierta edad que dices ahora los entiendo más

Hasta aquí llegó la cosa

Sí voy a llegar bien, aunque sea con la cabeza blanca
Va llegar un punto en que no me voy a encargar de ti
Va a llegar un cierto momento en la vida en que yo quiera bajarle al estrés

Va llegar un momento en que no me va encantar estar ahí

No sé qué tan lejos me gustaría llegar, porque la idea no es llegar si no cómo llegas [a la vejez]

Pero si ya llegas con limitaciones, físicas, por alguna enfermedad, eso sí me da miedo

En las metáforas regidas por el verbo llegar que se ejemplifican en los Cuadros 1, 2 y 3 se observa:

1) La estructura básica $(\mathrm{SN})+(\mathrm{Adv})+\mathrm{SV}+\mathrm{SP}$ para el foco en ego, como en (9):

(9) a. Yo ya iba a llegar a la meta.

b. Llegamos al fin del año. [Jimena, grupo 1]

Estudios de Lingüística Aplicada, año 35, número 66, diciembre de 2017, pp. 173-203 doi: 10.22201/enallt.01852647p.2017.66.836 
2) La estructura subordinante $(\mathrm{Adv})+\mathrm{SV}+\mathrm{SN}+\mathrm{SP}$ para el foco en time, como en (10):

(10) a. Va llegar un punto en que no me voy a encargar de ti. [Lorena, grupo 2]

b. Va llegar un momento en que no me va encantar estar ahí. [Marco, grupo 2]

3) Un par de casos en que la metáfora para el foco en time se encuentra en una estructura subordinada: SV + que $+\mathrm{SV}+\mathrm{SN}$. Ocurre cuando previamente en el discurso se habla de una etapa (fin de la universidad/jubilación) que termina co-referida en la palabra momento de la metáfora, como es el caso en (11) y (12):

(11) Astrid: Digamos, saliste de la universidad y ya de pronto estás así en un mundo más grande, y hay quien dice 'ay ya el mundo es mío' ¿no? [...] ¿Cómo te sientes? [...] ¿sobrecogido por ese mar de oportunidad o más como "ya llegué”? Raúl: O sea digo, yo creo que en un punto intermedio de los dos, mas no creo que sobrecogido, ni así como impactado, no sé o sea siento que era algo como que siempre, como que siempre sentía que iba llegar el momento y que pues ya, o sea que es normal, y que así. Que ya que como no es algo que me esté cogiendo por sorpresa [Raúl, grupo 1]

(12) Astrid: Por ejemplo, comparándonos ahora con la generación de nuestros papás, ¿tú qué harías diferente de ellos en cuanto a cuestiones de ahorro, cuestiones para la jubilación? No sé cómo está tu mamá ahora, ¿ella sigue trabajando?

Lucy: No, mi mamá ya no trabaja, mi papá sí. Pero ya mucho menos. Este...

Astrid: Es un esquema que ves como ejemplo, ¿o no te gustó?

Lucy: La verdad no me gustó porque personalmente siento que ellos no lo planearon bien. No sé si es porque pensaron que este momento no iba a llegar, no lo sé, pero a mí me queda muy claro que tengo que empezar a ahorrar desde ya porque no me gustaría tener miedo sobre no tener para comer o pagar la renta o ser dependiente de mi hija ¿no? [Lucy, grupo 2]

Hemos visto ya, a través del modelo que lo ilustra más arriba, cómo se integran conceptualmente tiempo y espacio en metáforas con el verbo llegar. La clave ha sido que los momentos y etapas de la vida pueden equipararse a lugares gra- 
cias a que las propiedades del verbo llegar son compatibles con muchos de los elementos del esquema LA VIDA ES UN CAMINO. Describo a continuación estas coincidencias.

El cambio es la clave que permite mapear los rasgos de un dominio a otro. Los aspectos de logro y de evento instantáneo son compatibles con el rasgo cambio de estado del dominio conceptual temporal. Este cambio está dado gracias a que el verbo codifica desplazamiento y permite perfilar la culminación del estado resultante que es estar en un lugar luego de haber cubierto dicha trayectoria. En todos los casos metafóricos que son objeto de análisis de este artículo, el resultado es aparecer en un momento de la vida. El lugar al que se llega no es una ubicación en el espacio sino en el tiempo, por ejemplo, llegar a los 40 años. El potencial de cambio contenido en llegar posibilita el acceso a ambos dominios, gracias a lo cual se pueden construir significados alrededor de este cambio.

Otro rasgo importante, además del cambio y el desplazamiento, es que el verbo llegar también codifica destino; en este caso, se trata del destino metafórico de una edad o etapa. El tiempo no solo modifica el estado de las cosas - como podemos observarlo en la decoloración de una tela, la oxidación de un metal o la maduración de una fruta — también nos modifica a nosotros. Cumplimos años y cambiamos de roles dentro de la sociedad. Dichos cambios de etapas son los destinos metafóricos del camino de la vida.

Precisamente, la metáfora LA VIDA ES UN CAMINO es compatible con el rasgo de trayectoria contenido también en llegar. El evento de la llegada es la culminación de dicho recorrido.

También hablé del trayector que recorre el camino. El verbo llegar requiere de una persona (agente) o momento (fuerza) que haga la acción.

Finalmente, el verbo puede codificar origen, aunque en este tipo de metáfora observo que este rasgo no se explicita en la instanciación. Sin embargo, es posible inferir un origen como ir de la década de los 30 a la de los 40 o del nacimiento a la muerte.

En cuanto a la perífrasis ir a +llegar, el aspecto prospectivo de la perífrasis también la vuelve compatible con el esquema del evento LLEGAR y con el mapeo espacio-temporal descrito. El rasgo más importante de la perífrasis, como afirma Bravo, es que describe situaciones conectadas con el presente, como en el ejemplo "No te sientes en esa roca. Se va a caer" en contraposición de "No te sientes en esa 
roca. Se caerá" (2008: 133). En palabras de Bravo: "Con 'va a + infinitivo', por consiguiente, lo que se localiza en la línea del tiempo no es propiamente el evento de caerse, sino el estado de cosas conducente a él. Cuando se emplea el futuro, en cambio, lo que se localiza es propiamente el evento de caerse la roca y no una situación anterior al mismo" (2008: 133). Por consiguiente, el aspecto prospectivo de la perífrasis focaliza también una fase previa al evento. La vinculación entre el estado de cosas que se afirma y una situación posterior es el rasgo que diferencia la perífrasis de la forma finita.

De acuerdo con la autora, hay además dos rasgos derivados de la aspectualidad prospectiva: un rasgo de intencionalidad del sujeto que realiza la acción del verbo auxiliado y un rasgo de inevitabilidad de la situación final anclada en el presente. En el caso de las metáforas de este artículo, ha sido interesante observar que cada rasgo es compatible con uno de los dos subesquemas de Boroditsky. El subesquema ego-moving contiene la noción de intencionalidad (véase (11) y (12)) en donde, si bien llegar a cierta edad es inevitable, la planificación para hacerlo de tal o cual manera implica una voluntad desde el presente. También se observa en el caso del papá de Jimena (ejemplo (13)) quien ya iba llegar a la meta pero lo regresaron al kínder y a las piñatas. En el entorno discursivo se construye la idea de que el padre ya estaba logrando cumplir el plan de la crianza y educación de las tres hijas universitarias y preparatorianas cuando volvió a tener un bebé.

(13) Astrid: ¿Tus papás todavía no se lo están planteando? [La jubilación]

Jimena: Nada, no, ahorita los veo como, y yo creo que tener un bebé los rejuveneció, creo, o sea bueno no sé, por lo menos 10,15 años van a seguir trabajando van a seguir haciendo, este...

Astrid: No y además hay que sacar todavía adelante, pues la colegiatura, educar, todavía es "papá y ve por mí a la fiesta de mi amiga ¿no?"

Jimena: Dice mi papá que es como el juego de serpientes y escaleras, yo ya iba a llegar a la meta y de repente me regresaron a las piñatas y al kínder, pero pues sí, o sea puede ser. [Jimena, grupo 1]

El rasgo de intencionalidad se observa en la perífrasis, tal como indica Bravo (2008). Además, en (13) la intencionalidad se ve coartada por lo que sucede 
después. De hecho se observa un rasgo que Bravo (2008) denomina "secundario" en la perífrasis porque solo se puede observar en ciertos contextos discursivos, como es este caso: la inminencia del evento de llegar a la meta. Dicha inminencia se ve anulada en esta narración.

En (14) se muestra la intencionalidad de la perífrasis, el lenguaje, adaptable como es a las situaciones más rígidas (Beckner, Blythe, Bybee, Christiansen, Croft, Ellis, Holland, Ke, Larsen-Freeman \& Schoenemann, 2009), se construye en torno a un hecho inexorable, por ejemplo, la vejez, pero focaliza la intención de otro de los sujetos, Yolanda, de llegar a esta en sus términos, habiendo disfrutado el recorrido:

(14) Pues lo veo mal. Bueno en sí, como que cuesta trabajo crecer y verte como grande. Ahorita que lo veo por ejemplo con mis abuelitos, con gente ya grande, es como de, yo voy a llegar a esa edad en algún momento de mi vida, pero pues es parte de la vida entonces, es algo normal, entonces es cuando te tienes que dar cuenta que debes de disfrutar cada momento, porque vas a llegar eso y vas a querer hacer todo lo que no hiciste pero no vas a poder, ya sea cuestión física, dinero o lo que quieras, pero, entonces algún momento llegaremos. [Yolanda, grupo 1]

Veamos ahora el rasgo de la perífrasis que propone Bravo (2008), la inevitabilidad, presente en el subesquema time-moving (ejemplos (10-12)). Dado que quien ejecuta la acción de llegar en este subesquema no es un agente, la intencionalidad no puede sostenerse en estos ejemplos. El tiempo no es un agente sino una fuerza que viene hacia el hablante. Sin embargo, el rasgo de inevitabilidad resulta totalmente compatible con este subesquema: no hay nada más inexorable que el advenimiento de los cambios de estado producidos por "el paso del tiempo". Todas las metáforas de la columna time-moving de los Cuadros 1, 2 y 3 expresan un sentido de inevitabilidad ante los eventos que mencionan: el final de la universidad, el cuerpo que ya no aguanta, el no poder ocuparse más de un ser querido, el deseo de modificar el ritmo de trabajo; todos ellos son traídos a la persona, lo quiera o no, por la fuerza del tiempo que llega. 


\subsection{Otros componentes de la metáfora}

Es importante señalar que otros componentes acompañan al verbo llegar en la construcción metafórica del tiempo, cuyas propiedades semánticas también son compatibles con los dominios temporal y espacial.

Un verbo de movimiento como llegar se acompaña de la preposición $a$, la cual tiene un rasgo locativo que establece una relación direccional entre los componentes sintácticos. La preposición $a$ también tiene un aspecto télico (Zwarts, 2006). Desde el esquema META-FUENTE-RUTA, pertenece naturalmente a la sección META (Demonte, 2011), por lo que el verbo llegar toma esta preposición para expresar el sitio de destino del evento. Es interesante observar que entre los ejemplos no están presentes otras preposiciones que acompañan al verbo llegar como de/desde para indicar procedencia, por para indicar trayecto ni en para indicar situación o vía.

Además, están los adverbios locativos deícticos. Este componente lingüístico pertenece al dominio conceptual espacial y aquí es empleado para referirse a un momento de la vida (dominio temporal). Las metáforas con adverbio deíctico locativo se dan en este subesquema ego-moving como se observó en el fragmento de discurso de Jimena (ejemplo (1)): O sea 90 es felicidades ¿cómo llegaste aquí? La persona sigue conceptualizando el cambio de edad como haber llegado a un lugar; en el discurso, y por ser esta la naturaleza del deíctico, dicho lugar se ha referido previamente: los 90 años. Dichas etapas de la vida se conceptualizan como espacios y por lo tanto se emplean deícticos locativos para referirlos.

Los rasgos locativos y télicos de la preposición $a$ y de los deícticos emergen en la instanciación lingüística pues son compatibles con el rasgo destino. Y dado que el destino es la culminación de un cambio de lugar, también mapean ese rasgo, compatible con cambio de estado.

Seguramente, para cada caso podrían identificarse rasgos semánticos que también ayudan al mapeo de conceptos espaciales al dominio de lo temporal, aunque he dado aquí un panorama general, enfocado al verbo llegar y a las menciones de los componentes presentes en la mayoría de los ejemplos. 


\section{CONCLUSIONES}

En este artículo analicé metáforas con el verbo llegar que conceptualizan el tiempo en términos de espacio, obtenidas en un espacio de interacción con sujetos entrevistados. Dichas metáforas emergieron en la charla que los sujetos entablaron conmigo para describir su trayecto de vida, sus diferentes etapas y cómo fueron transitando por algunas de ellas.

Un primer hallazgo ha sido que las formas más frecuentes de aparición del verbo llegar fueron la perífrasis ir a +infinitivo y las conjugaciones como núcleo del sv. Por lo tanto, son las dos formas que analicé en este trabajo.

En segundo lugar, observé que las metáforas se estructuran alrededor de dos subesquemas de la metáfora ontológica TIEMPO ES ESPACIO propuestas por Boroditsky (2000): ego-moving, en donde el individuo se conceptualiza como el agente que transita por un camino que representa un lapso determinado, y $t i$ me-moving, en donde el individuo se conceptualiza como el punto hacia el cual llegan los diferentes momentos y etapas de la vida, por lo que quien hace las veces de sujeto en este tipo de metáfora es una fuerza temporal.

En tercer lugar, los rasgos semánticos del verbo llegar, así como el aspecto prospectivo de la perífrasis y sus rasgos derivados, permiten mapear rasgos del dominio tiempo hacia el dominio espacio. El modelo de la integración conceptual permite proponer cómo es que esto ocurre. En el espacio genérico se coloca el rasgo cambio como la propiedad común que tiempo y espacio poseen entre sus características. El cambio de estado de las cosas hace patente el paso del tiempo en el dominio temporal; el significado de desplazamiento, codificado en el verbo llegar, pone en manifiesto el cambio de lugar en el dominio espacial. La forma finita y la perífrasis del verbo proporcionan además otros significados que ayudan a volver más concreta la metáfora TIEMPO ES ESPACIO, actualizándola a una versión asequible a escala humana: LA VIDA ES UN CAMINO.

Se observó en este análisis que no solo el rasgo cambio de estado se mapea de un dominio a otro gracias al componente verbal de la expresión, sino también gracias a otros componentes que refuerzan dicho mapeo: por ejemplo, la preposición $a$, que es consistente con el esquema metafórico específico de LA VIDA ES UN CAMINO, puesto que proporciona el rasgo de direccionalidad en el trayecto de vida del individuo. Los adverbios locativos ahí/aquí también refuerzan el 
mapeo de rasgos de espacio al dominio del tiempo gracias a su propiedad deíctica: en estas metáforas se refieren a momentos o edades de la vida y no a lugares. Dado que el rasgo cambio de estado es compatible con el rasgo cambio de lugar, ahí y aquí pueden operar en la integración de la instanciación lingüística.

Finalmente, es importante reflexionar sobre los alcances del modelo: la integración conceptual no abarca todos los rasgos conceptuales que explican la emergencia de una instanciación lingüística. Las expresiones que aquí se analizaron provienen de un discurso mucho mayor, producto de la interacción generada durante la entrevista semi-estructurada a los sujetos que participaron en la investigación. Muchos factores que al final dan origen a la instanciación linguiística, que en este caso concreto es una metáfora regida por el verbo llegar, se encuentran no solo en la instanciación sino también en secciones mayores de discurso.

El modelo de la integración conceptual proporciona una explicación parcial, razón por la cual propongo, en trabajos subsecuentes (Ruiz, 2018), una metodología de análisis que busca integrar las complejidades propias del lenguaje y del espacio de significación generado por la interacción de quienes participan en la entrevista.

\section{REFERENCIAS}

Arellanes, Francisco (2005). Los verbos de eventos instantáneos, los modificadores temporales y el copretérito en español: hacia una concepción composicional de la aspectualidad. En Martha Ruth Islas Canales \& Cynthia Araceli Ramírez Peñaloza (Comps.), Sintaxis del español e interfase sintaxis-semántica (pp.71-90). Toluca: Universidad Autónoma del Estado de México.

Beckner, Clay; Blythe, Richard; Bybee, Joan; Christiansen, Morten H.; Croft, William;

Ellis, Nick C.; Holland, John; Ke, Jinyun; Larsen-Freeman, Diane, \& Schoenemann, Том (2009). Language is a complex adaptive system: Position paper. En Nick C. Ellis \& Diane Larsen-Freeman (Eds.), Language as a complex adaptive system (pp. 1-26). Oxford: Blackwell.

BERgEN, BENJAMIN K. (2012). Louder than words: The new science of how the mind makes meaning. Nueva York: Basic Books.

BORODITSKY, LERA (2000). Metaphoric structuring: Understanding time through spatial metaphors. Cognition, 75(1), 1-28. 
Bravo Martín, ANA (2008). La perífrasis “ir a + infinitivo” en el sistema temporal y aspectual del español (Tesis doctoral inédita). Universidad Complutense de Madrid, Madrid. Recuperado de http://eprints.ucm.es/8074/

Demonte, Violeta (2011). Los eventos de movimiento en español: construcción léxico-sintáctica y microparámetros preposicionales. En Juan Cuartero Otal, Luis García Fernández \& Carsten Sinner (Coords.), Estudios sobre perífrasis y aspecto (pp. 16-42). Múnich: Peniope.

FAuconnier, Gilles (1984). Espaces mentaux: Aspects de la construction du sens dans les langues naturelles. París: Les Éditions de Minuit.

Fauconnier, Gilles (1997). Mapping in thought and language. Cambridge: Cambridge University Press.

FAuconnier, Gilles, \& Turner, Mark (2002). The way we think: Conceptual blending and the mind's hidden complexities. Nueva York: Basic Books.

Fedden, Sebastian, \& Boroditsky, Lera (2012). Spatialization of time in Mian. Frontiers in Psychology, 3(19), 1-9.

GARCía FERnÁndez, LuIs (2000). La gramática de los complementos temporales. Madrid: Visor.

Genta, Florencia (2008). Perífrasis verbales en español: focalización aspectual, restricción temporal y rendimiento discursivo (Tesis doctoral inédita). Universidad de Granada, Granada.

Hidalgo Navarro, Antonio (1997). Sobre los mecanismos de impersonalización en la conversación coloquial: el tú impersonal. Estudios de Lingüística, 11, 163-176.

InSTITUTO NACIONAL DE Estadística y GEOGRAFía, INEGI (2001). Indicadores sociodemográficos de México (1930-2000). Aguascalientes: INEGI. Recuperado de http://www.beta.inegi.org.mx/ app/biblioteca/ficha.html?upc $=702825493875$

Kövecses, Zoltan (2002). Metaphor: A practical introduction. Oxford: Oxford University Press.

Lakoff, George, \& Johnson, Mark (1986 [1980]). Metáforas de la vida cotidiana. Madrid: Cátedra.

LANGacker, Ronald Wayne (1987). Foundations of cognitive grammar: Theoretical prerequisites (Vol. 1). Stanford: Stanford University Press.

Montes de Oca Zavala, Verónica (2010). Pensar la vejez y el envejecimiento en el México contemporáneo. Renglones, 62, 159-181.

NúÑez, RAfael, \& CoOperrider, Kensy (2013). The tangle of space and time in human cognition. Trends in Cognitive Science, 17(5), 220-229.

Núñez, Rafael; Neumann, Vicente, \& Mamani, Manuel (1997). Los mapeos conceptuales en la concepción del tiempo en la lengua aymará del norte de Chile. Boletín de Educación de la Universidad Católica del Norte, 28(21), 47-55.

Estudios de Lingüística Aplicada, año 35, número 66, diciembre de 2017, pp. 173-203

doi: 10.22201/enallt.01852647p.2017.66.836 
Ruiz Surget, AleXANDRA Astrid (2011). La construcción metafórica-discursiva del rostro como parte por el todo de la identidad: dos estudios de caso del francés y el español (Tesis de maestría inédita). Universidad Nacional Autónoma de México, Ciudad de México.

Ruiz Surget, Alexandra Astrid (2018). Procesos de conceptualización del tiempo en términos de espacio: hablar del tiempo-espacio de la vida. México: Universidad Nacional Autónoma de México.

VAn Dijk, Teun Adrianus (1999). Ideología: un enfoque multidisciplinario. Barcelona: Gedisa.

VAn Dijk, Teun Adrianus (2001). Algunos principios de una teoría del contexto. Revista Latinoamericana de Estudios del Discurso, 1(1), 69-81.

Vendler, Zeno (1957). Verbs and times. The Philosophical Review, 2(66), 143-160. Recuperado de http://semantics.uchicago.edu/scalarchange/vendler57.pdf

Wilson, Nicole L., \& GibBS JR., RAYMOND W. (2007). Real and imagined body movement primes metaphor comprehension. Cognitive Science: A Multidisciplinary Journal, 31(4), 721-731.

Wittmann, Marc (2013). The inner sense of time: How the brain creates a representation of duration. Neuroscience, 14, 217-223.

Zwarts, Joost (2006). Event shape: Paths in the semantics of verbs. Nimega: Ms. Radboud University Nijmegen. Recuperado de http://www.hum.uu.nl/medewerkers/j.zwarts/EventShape.pdf 
